\section{Ensayo clínico controlado de superovulación e inseminación por infertilidad asociada con endometriosis mínima o leve}

Ian S. Tummon, M.D. $\dagger \ddagger$

Linda J. Asher, R.N.C. $\dagger$

James S.B. Martín, M.D.†

Togas Tulandi, M.D.§

+ Department of Obstetrics and Gynaecology, The University of Western Ontario.

† Department of Gynaecology and Reproductive Medicine, London Health Sciences Centre, University Campus.

\section{§Department of Obstetrics and Gynaecology, McGill University.}

Fertil Steril 1997; 68: 8-12.

Objetivo: Evaluar la eficacia de la superovulación e inseminación intrauterina (IIU) versus no tratamiento para la infertilidad asociada con la endometriosis mínima o leve.

Diseño: Ensayo aleatorizado

Lugar: Centro de ciencias de la salud de Londres, campo universitario, Universidad de Western Ontario, Londres, Ontario y Hospital Royal Victoria, Universidad de McGill, Montreal, Quebec, Canadá.

Pacientes: Trescientos once ciclos en 103 parejas en quienes la endometriosis mínima o leve fue el único factor de subfertilidad identificado.

Intervención: superovulación con FSH e IIU.

Medida principal de resultado: Nacidos vivos.

Resultados: Se obtuvieron nacidos vivos en 14 de $127(11 \%)$ ciclos de superovulación e IIU y en 4 de 184 (2\%) de los ciclos de no tratamiento. El Odds ratio fue 5.6 (intervalo de confianza del $95 \% 1.8$ a 17.4) a favor de la superovulación y la IIU.

Conclusiones: El tratamiento con superovulación e IIU se asoció con un resultado superior en las tasas crudas de recién nacidos vivos y en el análisis proporcional de riesgo.

\section{Dolor pélvico asociado con endometriosis: eviden- cia para una asociación entre el estado de la enfer- medad y una historia de dolor pélvico crónico}

Dale W. Stovall, M.D. $\dagger \ddagger$

Lisa M. Bowser, B.S.§

David F. Archer, M.D.I

David S. Guzick, M.D.†I
+ Division of Reproductive Endocrinology, Department of Obstetrics and Gynecology. University of Pittsburgh School of Medicine.

$\$$ Department of Obstetrics and Gynecology, Division of Reproductive Endocrinology. University of Iowa School of Medicine.

$\S$ Department of Physician Assistant, Duquesne University.

$\backslash$ Department of Obstetrics and Gynecology, Eastern Virginia School of Medicine.

II Present address: Department of Obstetrics and Gynecology. University of Rochester, Rochester, New York.

Fertil Steril 1997; 68: 13-18.

Objetivo: Investigar la severidad y localización del dolor pélvico asociado con endometriosis durante los años de vida reproductiva y evaluar la asociación entre estos parámetros de dolor y el estado de la enfermedad.

Diseño: Estudio histórico prospectivo.

Lugar: Centro de cuidado terciario.

Pacientes: cuarenta y ocho mujeres con endometriosis clasificada endoscópicamente y dolor pélvico crónico quienes fueron sometidas a tratamiento médico o quirúrgico conservador.

Intervención: A cada paciente se le suministró un cuestionario que incluía una determinación de la localización y la severidad de su dolor.

Medidas principales de resultado: Fueron registrados el estado de la enfermedad, el área de la pelvis que contenía la enfermedad, la severidad del dolor y la localización del sitio de dolor mas severo.

Resultados: La duración promedio desde el diagnóstico inicial hasta el seguimiento fue de $15.7 \pm 3.1$ años. Veintiún $(43.8 \%)$ sujetos negaron cualquier síntoma de dolor durante la evaluación de seguimiento. De las 27 pacientes con dolor persistente, $21(78 \%)$ identificaron la localización de su dolor mas severo como la misma que en el diagnóstico inicial. El estado de la enfermedad en el momento del diagnóstico estuvo significativamente asociado con un mayor grado de dolor en el seguimiento.

Conclusión: Estos datos sugieren que el dolor pélvico asociado con la endometriosis comúnmente persiste durante la vida reproductiva y que el estado de la enfermedad está directamente relacionado con la persistencia del dolor pélvico. 